This item was submitted to Loughborough's Research Repository by the author.

Items in Figshare are protected by copyright, with all rights reserved, unless otherwise indicated.

\title{
Experimental investigation of the Kelvin-Helmholtz instabilities of cylindrical gas columns in viscous fluids
}

PLEASE CITE THE PUBLISHED VERSION

https://doi.org/10.1016/j.ijmultiphaseflow.2018.03.017

\section{PUBLISHER}

(C) Elsevier

VERSION

AM (Accepted Manuscript)

\section{PUBLISHER STATEMENT}

This work is made available according to the conditions of the Creative Commons Attribution-NonCommercialNoDerivatives 4.0 International (CC BY-NC-ND 4.0) licence. Full details of this licence are available at: https://creativecommons.org/licenses/by-nc-nd/4.0/

\section{LICENCE}

CC BY-NC-ND 4.0

\section{REPOSITORY RECORD}

Zhao, Huayong, and Benjamin Bhabra. 2019. "Experimental Investigation of the Kelvin-helmholtz Instabilities of Cylindrical Gas Columns in Viscous Fluids". figshare. https://hdl.handle.net/2134/32545. 


\section{Title: Experimental Investigation of the Kelvin-Helmholtz Instabilities of}

\section{Cylindrical Gas Columns in Viscous Fluids}

Abbreviated title: Hydrodynamic Instability in Viscous Fluid

Huayong Zhao $^{1^{*}}$, Benjamin Bhabra ${ }^{1}$

1. Wolfson School of Mechanical, Electrical and Manufacturing Engineering Loughborough University, Leicestershire, LE11 3TU, United Kingdom

* Corresponding author. Tel: +44(0)1509227530; email: $\underline{\text { H.Zhao2@lboro.ac.uk }}$

Co-author email address: B.J.Bhabra-14@student.lboro.ac.uk

\section{Abstract}

This paper derives analytical solutions for the critical Kelvin-Helmholtz $(\mathrm{KH})$ instability conditions at the interface between a cylindrical gas column and a pool of viscous immiscible fluid confined in a chamber of finite size. The analysis focuses on conditions of negligible heat and mass transfer. The derivations are based on the established approaches reported in the literature with different boundary conditions. The most unstable instability conditions have also been calculated numerically. Experiments designed to measure the actual air column break-up conditions in water have been carried out to validate the analytical models. Comparisons show that the most unstable conditions predicted by the Viscous Corrections of the Viscous Potential Flow KH model are the best match to the experimentally measured break-up conditions. Parametric investigation of the instability theories shows that the vapour column size has a noticeable effect on the critical conditions, but has a negligible effect 
on the most unstable conditions when the column radius is greater than 1.2 $\mathrm{mm}$. Furthermore, the critical instability conditions are sensitive to the chamber size and the perturbation symmetry, while the most unstable conditions are insensitive to these parameters.

Keywords: Kelvin-Helmholtz instabilities; viscosity; cylindrical interface.

\section{Introduction}

Hydrodynamic instabilities in gas-liquid systems play significant roles in a wide range of applications in chemical engineering and geophysics. For example, in boiling heat transfer, the Kelvin-Helmholtz $(\mathrm{KH})$ instability has been widely adopted to explain and predict the occurrence of the critical heat flux condition (i.e. the maximum heat flux point at the end of the nucleation boiling regime), while the Rayleigh-Taylor (RT) instability has been applied to predict the heat transfer rate in film boiling regimes (Carey, 2008).

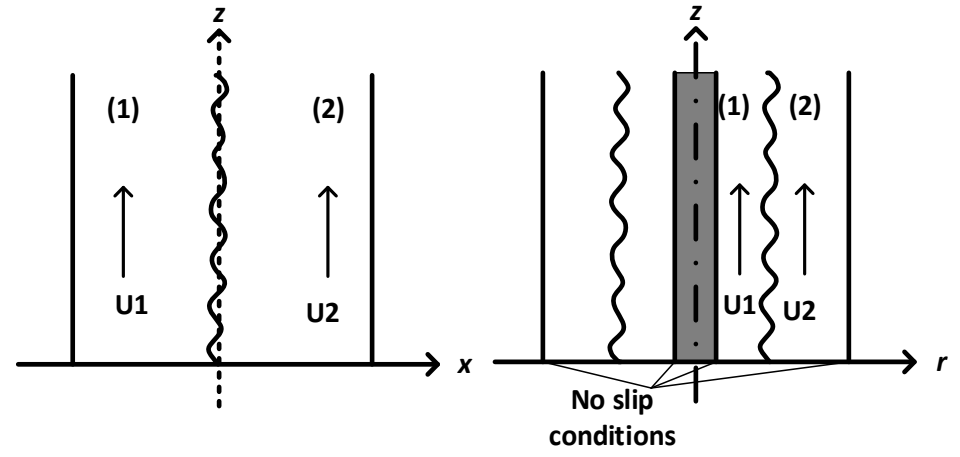

(a) (b)

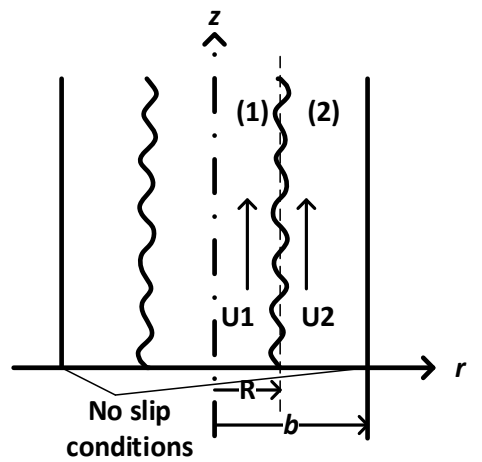

(c)

Fig. 1 Different geometries of interfaces and boundary conditions reported in the literature

The widely adopted $\mathrm{KH}$ and RT instability theories in boiling heat transfer, as summarized in the literature (Carey, 2008; Zhao and Williams, 2018), are based 
on the linear Inviscid Potential Flow (IPF) analysis of a planar interface between two infinitely deep pools of inviscid fluid without interfacial heat and mass transfer (Fig. 1 (a)). Hsieh (1978) reported the first corrections to the planar interface stability and interfacial heat and mass transfer due to the finite thickness of the gas and liquid layers. Nayak and Chakraborty (1984) later adopted the methodology developed by Hsieh to analyse a cylindrical interface with heat and mass transfer, as shown in Fig. 1 (b), a configuration which mimics the cooling of fuel rods in nuclear reactors. They did not derive the explicit solutions but instead solved the dispersion equation numerically. The nonlinear IPF analysis of the KH instability of a cylindrical interface, as in Fig.1 (b), with heat and mass transfer, has been carried out by Lee (2007). Lee provided the explicit solution for the linear instability criteria and the numerical solutions for the higher-order nonlinear analysis.

Although the IPF assumptions greatly simplify the analysis, neglecting the effect of viscosity limits its accuracy. Funada and Joseph (2001) introduced the first correction for the effect of viscosity by carrying out the so-called 'Viscous Potential Flow' (VPF) analysis on a planar interface without heat and mass transfer, as shown in Fig. 1 (a). Their analysis considers the normal shear stress without violating the assumption of irrotational flow and only considers the linear instability term. A comparison of the results predicted by VPF and IPF analyses suggests that the critical values of the relative velocities between the air and liquid are independent of the viscosity when the liquid viscosity is $>15$ $\mathrm{mPa} \cdot \mathrm{s}$. However, when the liquid viscosity is $<15 \mathrm{mPa} \cdot \mathrm{s}$, the critical velocity predicted by VPF analysis is lower than that of IPF analysis. Their analysis also 
showed that the critical relative velocity was smaller in a thinner gas layer. A similar VPF analysis has been applied to solve the first-order $\mathrm{KH}$ instabilities of the cylindrical interface around a solid fuel rod, as shown in Fig.1 (b) (Awasthi and Agrwal, 2011; Asthana, Awasthi and Agrawal, 2014). Their analysis showed that the viscosity reduced the instability growth rate, but they did not compare the required critical velocity predicted by the IPF and VPF analyses. Recently, Awasthi et al. (2016) applied a similar VPF approach (i.e. taking into account the normal shear stress without violating the irrotational flow assumption) to investigate the non-linear $\mathrm{KH}$ instability of a cylindrical interface with heat and mass transfer, as in Fig. 1 (b). To test the accuracy of the VPF theory, Funada and Joseph (2002) compared the capillary instability criteria predicted by the VPF theory and the Fully Viscous Flow (FVF) theory for a liquid cylinder at rest. The FVF theory is the exact normal mode solution of the linearized Navier-Stokes equations. They calculated the instability growth rates at different Reynolds numbers, which were defined by the capillary velocity (i.e. $R e=\sigma D \rho_{l} / \mu_{l}^{2}$, where $\sigma$ is the surface tension, $\rho_{l}$ is the liquid density, $\mu_{l}$ is the liquid dynamic viscosity and $D$ is the fluid column diameter). The equations of the FVF theory were solved numerically, while the VPF model has an explicit solution for the critical instability condition. Their results showed that the VPF analysis gave good approximations to the FVF results when the Reynolds number was $>O\left(10^{1}\right)$ (i.e. orders of magnitude of $10^{1}$ ) and was always more accurate than the IPF theory. To further correct the viscous shear force while still using the potential flow analysis, Joseph and Wang (2004) developed a dissipation approximation theory, named the 'Viscous Correction of the Viscous 
Potential Flow' (VCVPF) theory. The VCVPF approach introduced a viscous pressure term to resolve the discrepancy between the zero shear stress boundary condition at a free surface and the non-zero irrotational shear stress. The VCVPF approach has been applied to analyse the capillary instability of a cylindrical liquid column in air by Wang et al. (2005). They showed that the VCVPF method provided more accurate predictions of the instability growth rates than IPF and VPF models. The VCVPF theory has also been used to analyse the linear KH instability for both a planar (Fig. 1 (a)) and a cylindrical interface around a solid fuel rod (Fig.1 (b)) with heat and mass transfer under both axisymmetric and asymmetric disturbance (Awasthi, Asthana and Agrawal, 2012, 2014). Awasthi et al. $(2012,2014)$ derived the explicit solutions for the instability criteria, and their calculated results showed that the VCVPF solution was more stable than the VPF solution. In addition, the instability growth rate was found to be smaller for the asymmetric disturbance conditions when compared to the symmetric disturbance conditions. However, there was no direct comparison of the neutral curves (i.e. the critical relative velocity at different instability wavelengths) predicted by the IPF, VPF and VCVPF models.

In summary, compared to the IPF and VPF theories, the VCVPF theory has been shown to be a more accurate approximation to the FVF solution for predicting the capillary instability of a cylindrical liquid column on an infinite liquid reservoir with an axisymmetric disturbance. However, in practical systems -- such as in boiling and in fluidised beds -- the perturbation can hardly be strictly axisymmetric, and the instability can be induced not only by the capillary force but also by the velocity difference due to forced convection. The effects of 
symmetry and interfacial velocity on the instability of the two-phase flow around a cylindrical fuel rod, as shown in Fig. 1 (b), have been analysed theoretically by Awasthi et al. (2012, 2014). However, there are no direct experimental data to test the validity of these solutions. Moreover, no analytical solutions can be found to predict the $\mathrm{KH}$ instability at the interface between a finite-sized cylindrical gas column and a pool of viscous liquid confined in a finite-sized chamber (Fig. 1 (c)), as in typical boiling systems. Furthermore, there is still a debate on whether the break-up of a gas column occurs more consistently in the critical condition (i.e. when the instability just starts to grow with time) or in the most unstable condition (i.e. when the instability growth rate is highest) due to the lack of direct experimental data.

In this paper, the IPF, VPF and VCVPF solutions of the $\mathrm{KH}$ instability for a cylindrical interface shown in Fig. 1 (c), with negligible heat and mass transfer across the interface, will be presented. These solutions will be compared to the experimental data gathered from a gas blowing rig. Finally, a detailed comparison and parametric analysis of the IPF, VPF and VCVPF solutions for both the critical condition and the most unstable condition will be reported and discussed.

\section{Formulation of the Kelvin-Helmholtz (KH) Instability}

The $\mathrm{KH}$ instability occurs when there is a velocity difference across the interface between two fluids. The analysis presented here is based on a vertical interface between a finite-sized cylindrical column occupied by fluid 1 and a cylindrical finite-sized reservoir occupied by fluid 2, as shown in Fig. 1 (c). Cylindrical 
coordinates $(r, \theta, z)$ are used for the analysis. When in equilibrium, fluid 1 occupies the space between $r=0$ and $r=R$, while fluid 2 occupies the space between $r=R$ and $r=b$. The properties of fluid 1 are labelled with the subscript 1 (e.g. density - $\rho_{1}$, dynamic viscosity - $\mu_{1}$, flow velocity - $U_{1}$ and thermal conductivity $-k_{1}$ ), while the properties of fluid 2 are labelled with the subscript 2.

The instability growth rates can be derived from the normal model analysis, as detailed in Appendix 1, together with the following boundary conditions:

(1) No slip condition on the rigid chamber wall.

(2) Finite value of the velocity in the central axis.

Following the IPF approach, the following dispersion equation, which is similar to the one suggested by Lee (2007), can be derived:

$a_{0} w^{2}+a_{1} w+a_{2}=0$

$a_{0}=\rho_{2} E_{1 R}-\rho_{1} F_{1 R} ; a_{1}=2 k\left(U_{1} \rho_{1} F_{1 R}-U_{2} \rho_{2} E_{1 R}\right)$

$a_{2}=k^{2}\left(U_{2}^{2} \rho_{2} E_{1 R}-U_{1}^{2} \rho_{1} F_{1 R}\right)+\frac{k \sigma}{R^{2}}\left(k^{2} R^{2}-1+m^{2}\right) ;$

$E_{1 R}=E_{1}(k R) ; F_{1 R}=F_{1}(k R) ; E_{1}(k r)=\frac{I_{m}(k r) K_{m}^{\prime}(k b)-K_{m}(k r) I_{m}^{\prime}(k b)}{I_{m}^{\prime}(k R) K_{m}^{\prime}(k b)-I_{m}^{\prime}(k b) K_{m}^{\prime}(k R)} ; F_{1}(k r)=\frac{I_{m}(k r)}{I_{m}^{\prime}(k R)}$

where $k$ is the instability wavenumber and is correlated to the instability wavelength $(\lambda)$ by $k=2 \pi / \lambda$;

$w=w_{r}+i w_{i}$ is the complex growth rates;

$I_{m}$ is the modified Bessel function of the first kind; 
$K_{m}$ is the modified Bessel function of the second kind;

$m$ is the symmetric index along the $\theta$ direction.

The function $F_{1 R}$ in the solutions derived by Lee (2007) is different from that in Eq. (1) due to the different boundary conditions. Substituting $w$ in Eq. (1) with $w_{r}+i w_{i}$, the following equation can be derived to solve for $w_{i}$ to determine whether the instability will grow or decay:

$4 a_{0}^{3} w_{i}^{4}-\left(4 a_{0}^{2} a_{2}-a_{0} a_{1}^{2}\right) w_{i}^{2}=0$

The most unstable condition corresponds to the condition where $w_{i}$ reaches its maximum value. Both the most unstable wavelength and the instability growth rate for different relative velocities can be calculated from Eq. (2).

The critical condition (i.e. $w_{i}=0$, where the instability is neither growing or deteriorating, often referred to as 'the neutral curve' in the literature), as is shown in Eq. (3), can be obtained based on the condition: $a_{1}^{2}-4 a_{0} a_{2}=0$, i.e.,

$V_{I P F}^{2}=\left(U_{1}-U_{2}\right)^{2}=\frac{\sigma}{k R^{2}}\left(1-k^{2} R^{2}-m^{2}\right) \cdot \frac{\rho_{1} F_{1 R}-\rho_{2} E_{1 R}}{\rho_{1} \rho_{2} E_{1 R} F_{1 R}}$

where $V$ is the relative velocity between fluid 1 and fluid 2 .

In comparison, the inviscid $\mathrm{KH}$ instability theory used in the existing boiling critical heat flux model is based on the planar vertical interface shown in Fig. 1 (a). This theory will be referred to as the 'Planar Inviscid Potential Flow' (PIPF) theory in this paper, and it takes the following form (Carey, 2008):

$V_{I P F-p}^{2}=\left(U_{1}-U_{2}\right)^{2}=\sigma k \cdot \frac{\rho_{1}+\rho_{2}}{\rho_{1} \rho_{2}}$ 
The most unstable condition predicted by the PIPF can be calculated by Eq. (5), which is derived by taking $\partial w_{i} / \partial k=0$ to find the maximum growth rate condition, viz:

$V_{I P F-p}^{2}=\left(U_{1}-U_{2}\right)^{2}=\frac{3 \sigma k}{2} \cdot \frac{\rho_{1}+\rho_{2}}{\rho_{1} \rho_{2}}$

Following the VPF approach (i.e. taking into account the pressure term caused by the normal viscous force), the dispersion equation can be derived as:

$$
\begin{aligned}
& a_{0} w^{2}+\left(a_{1}+i b_{1}\right) w+\left(a_{2}+i b_{2}\right)=0 \\
& a_{0}=\rho_{2} E_{1 R}-\rho_{1} F_{1 R} ; a_{1}=2 k\left(U_{1} \rho_{1} F_{1 R}-U_{2} \rho_{2} E_{1 R}\right) ; \\
& b_{1}=2 k^{2}\left(\mu_{2} E_{2 R}-\mu_{1} F_{2 R}\right) ; a_{2}=k^{2}\left(U_{2}^{2} \rho_{2} E_{1 R}-U_{1}^{2} \rho_{1} F_{1 R}\right)+\frac{k \sigma}{R^{2}}\left(k^{2} R^{2}-1+m^{2}\right) ; \\
& b_{2}=2 k^{3}\left(\mu_{1} U_{1} F_{2 R}-\mu_{2} U_{2} E_{2 R}\right) ; \\
& E_{2 R}=E_{1 \mathrm{R}}\left(1+\frac{m^{2}}{k^{2} R^{2}}\right)-\frac{1}{k R} ; F_{2 R}=F_{1 \mathrm{R}}\left(1+\frac{m^{2}}{k^{2} R^{2}}\right)-\frac{1}{k R}
\end{aligned}
$$

The critical instability condition can be found when $a_{0} b_{2}^{2}-a_{1} b_{1} b_{2}+a_{2} b_{1}^{2}=0$, that is, where

$$
V_{V P F}^{2}=\left(U_{1}-U_{2}\right)^{2}=\frac{\left[\sigma k\left(k^{2} R^{2}+m^{2}-1\right) / R^{2}\right] \cdot\left[2 k^{2}\left(\mu_{1} F_{2 R}-\mu_{2} E_{2 R}\right)\right]^{2}}{4 k^{6}\left(\rho_{1} \mu_{2}^{2} F_{1 R} E_{2 R}^{2}-\rho_{2} \mu_{1}^{2} E_{1 R} F_{2 R}^{2}\right)}
$$

The functions $F_{1 R}$ and $F_{2 R}$ in the solutions reported by Awasthi and Agrwal (2011) are different from those in Eqs. (6) and (7) due to the different boundary conditions.

Following the VCVPF approach introduced by Wang et al. (2005), the following dispersion equation can be derived: 
$a_{0} w^{2}+\left(a_{1}+i b_{1}\right) w+\left(a_{2}+i b_{2}\right)=0$

$a_{0}=\rho_{2} E_{1 R}-\rho_{1} F_{1 R} ; a_{1}=2 k\left(U_{1} \rho_{1} F_{1 R}-U_{2} \rho_{2} E_{1 R}\right) ;$

$b_{1}=2 k^{2}\left[\mu_{2} E_{3 R}-\mu_{1} F_{3 R}\right] ; a_{2}=k^{2}\left(U_{2}^{2} \rho_{2} E_{1 R}-U_{1}^{2} \rho_{1} F_{1 R}\right)+\frac{k \sigma}{R^{2}}\left(k^{2} R^{2}-1+m^{2}\right) ;$

$b_{2}=2 k^{3}\left[\mu_{1} U_{1} F_{3 R}-\mu_{2} U_{2} E_{3 R}\right] ; E_{3 R}=E_{1 R}+E_{2 R} ; F_{3 R}=F_{1 R}+F_{2 R}$

The neutral curve can be found based on the condition $a_{0} b_{2}^{2}-a_{1} b_{1} b_{2}+a_{2} b_{1}^{2}=$ 0 , as in Eq. (9).

$V_{V C V P F}^{2}=\left(U_{1}-U_{2}\right)^{2}=\frac{\left[\sigma k\left(k^{2} R^{2}+m^{2}-1\right) / R^{2}\right] \cdot\left[2 k^{2}\left(\mu_{1} F_{3 R}-\mu_{2} E_{3 R}\right)\right]^{2}}{4 k^{6}\left(\rho_{1} \mu_{2}^{2} F_{1 R} E_{3 R}^{2}-\rho_{2} \mu_{1}^{2} E_{1 R} F_{3 R}^{2}\right)}$

The functions $F_{1 R}, F_{2 R}$ and $F_{3 R}$ in the solutions derived by Awasthi et al. (2014) are different from those in Eqs. (8) and (9) due to the different boundary conditions.

\section{Experimental Setup and Data Processing}

Experiments designed to measure the actual vapour column break-up conditions where there is negligible heat and mass transfer have been conducted to validate different $\mathrm{KH}$ instability models. The experimental setup is shown in Fig. 2. It includes a transparent water chamber, an air chamber connected to an air delivery line, a perforated plate sealed between the water and air chambers, and control and measurement instruments. The key premise is to feed the air through the perforated plate into the water chamber, and quantify the air column break-up conditions through a high-speed backlit shadowgraph imaging system. The imaging system consists of two frame-byframe synchronised high-speed cameras. The water chamber and air chamber 
are made of transparent acrylic tubes of $76.7 \mathrm{~mm}$ outer diameter and $3.2 \mathrm{~mm}$ wall thickness. The perforated plates are changeable, and they have different hole patterns. Four different hole patterns, which consist of a series of $0.5 \mathrm{~mm}$ diameter holes distributed uniformly in areas of different diameter at $0.5 \mathrm{~mm}, 2$ $\mathrm{mm}, 5.6 \mathrm{~mm}$ and $10.2 \mathrm{~mm}$, were used in tests.

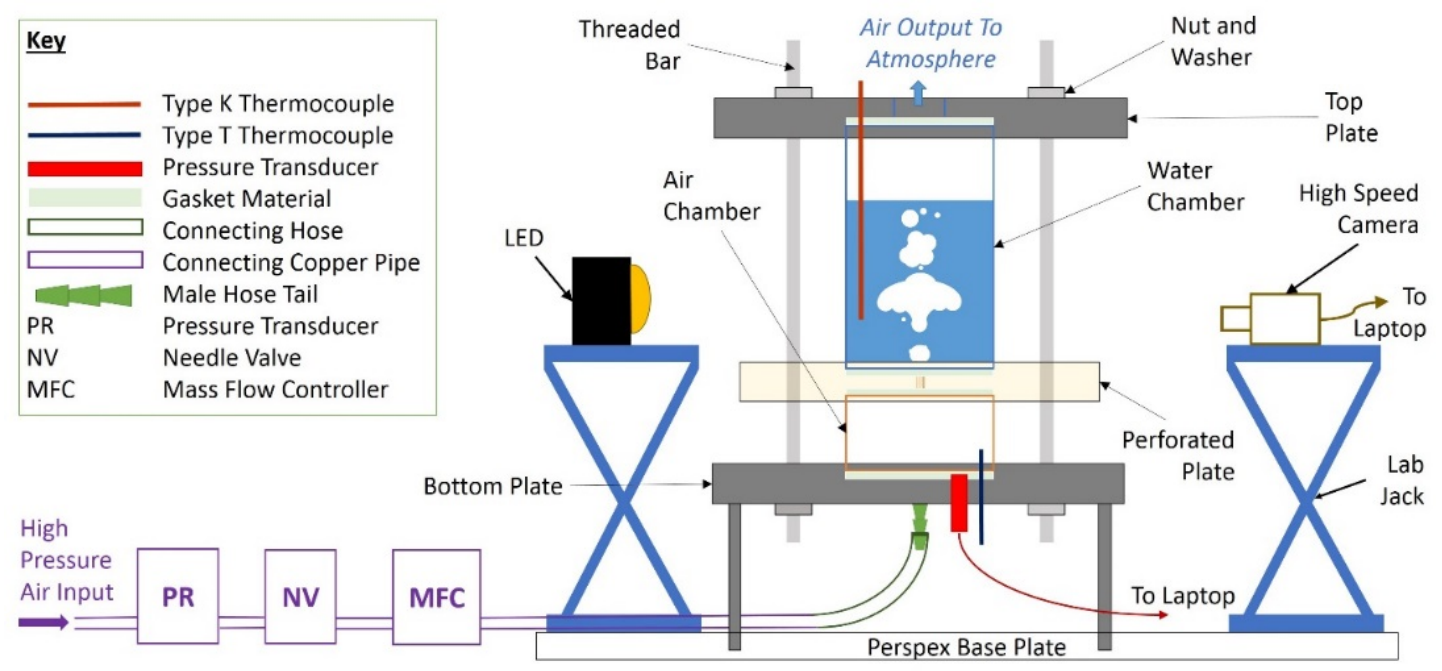

Fig. 2 Schematic experimental setup of the vapour column instability rig

The air delivery line, which consists of a high-pressure air feed, a pressure regulator, a needle valve and a mass flow controller, can deliver up to $10 \mathrm{l} / \mathrm{min}$ air flow. Leak tests using a water-based leak detector were undertaken before each test to ensure there was no air leakage.

An absolute pressure transducer and a type $\mathrm{T}$ thermocouple were fitted to the bottom plate to monitor the air chamber pressure and temperature. The top of the water chamber was left open to the atmosphere and was filled with purified water up to a depth of $10 \mathrm{~cm}$. The temperature of the water was monitored by a Type $\mathrm{K}$ thermocouple immersed in the water to ensure there was a negligible temperature difference between the air and the water. 
A series of tests, which were set under different flow conditions and using different perforated plates, were conducted at the conditions summarised in Table 1.

Table 1 Summary of experimental conditions

\begin{tabular}{|l|l|l|l|l|}
\hline & Plate 1 & Plate 2 & Plate 3 & Plate 4 \\
\hline $\begin{array}{l}\text { Flow rate range } \\
(l / \text { min })\end{array}$ & $1-3$ & $1-5$ & $1-8$ & $6-8$ \\
\hline $\begin{array}{l}\text { Air chamber pressure } \\
\text { (bar, absolute) }\end{array}$ & $1.04-1.13$ & $1.03-1.04$ & $1.02-1.03$ & 1.022 \\
\hline
\end{tabular}

Before each test, both cameras were calibrated against a calibration target to align their imaging centres and calibrate their magnifications. The two cameras were both set at 2000 frames per second and $1280 \times 800$ pixel resolution to capture the detailed air column break-up processes. The generated pairs of images were then digitally cropped and enhanced before being used to reconstruct the three-dimensional structures of the air column using an algorithm developed by Sechenyh et al. (2017), as shown in Fig. 3.
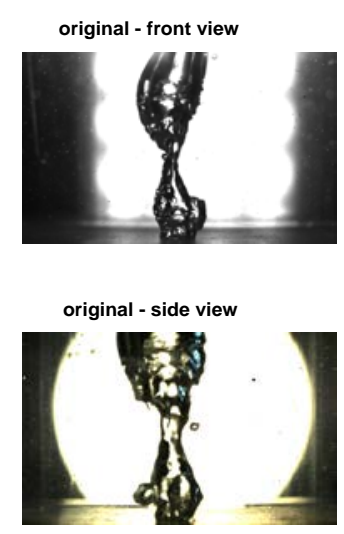

(a) Original

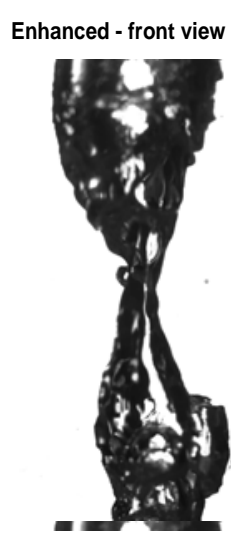

(b) Cropped and enhanced
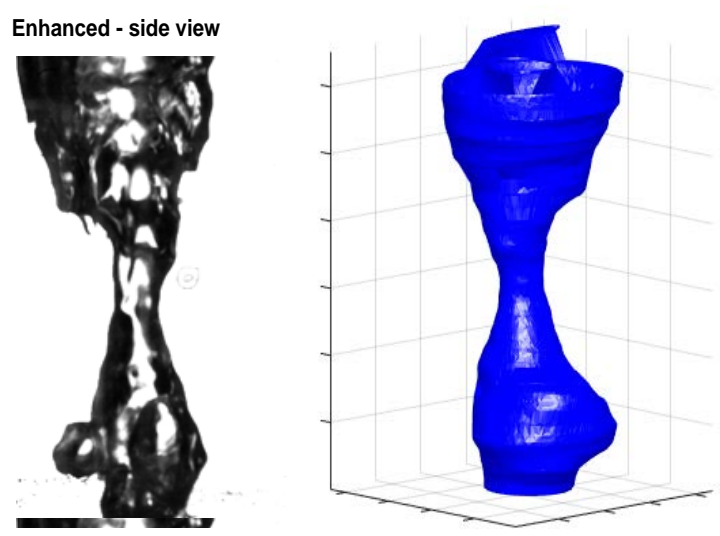

(c) Reconstructed

Fig. 3 The air column necking process shown from two orthogonal angles. The cross-sectional area and the height of the break-up position were then obtained from the reconstructed air column when it just starts to neck before 
breaking up. Although the air column was subjected to perturbations of different wavelengths, it was assumed to break-up at heights equivalent to the dominant wavelength predicted by the $\mathrm{KH}$ instability, i.e. $h_{\text {neck }}=\lambda$. The air velocity was then calculated as the air volume flow rate divided by the measured crosssectional area. The measured height of the necking position was compared to both the critical instability wavelength and the most unstable instability wavelength predicted by different $\mathrm{KH}$ instability models to determine which was most accurate when applied to practical systems. The air mass flow rate controller was calibrated to have an error of within $1 \%$. The camera and optical system can achieve a resolution of better than $10 \mu \mathrm{m}$. The errors in the measurement of the dimensions were mainly due to the reconstruction algorithm that only utilizes two orthogonal views (i.e. assuming an elliptical cross-section) and is thus dependent on the shapes of the vapour column. The original paper that introduced this algorithm (Sechenyh et al., 2017) suggested that the uncertainty should be less than $5 \%$ for objects with smooth surfaces and up to $10 \%$ for objects with complex surface features.

\section{Results and Discussion}

The critical and the most unstable instability conditions predicted by different $\mathrm{KH}$ instability theories are presented in this section together with the measured break-up conditions. The results from the parametric analysis of different $\mathrm{KH}$ models are also shown in this section to demonstrate the effects of vapour column size, water chamber size and perturbation symmetry on the instability conditions. 


\subsection{Instability of the Interface with Axisymmetrical Perturbation}

The air and water properties used in the calculations are listed in Table 2. The liquid velocity is assumed to be negligibly small compared to the air velocity. In practical systems, the air column interface is subjected to perturbations of different instability wavelengths. Among these, only the perturbations at wavelengths larger than the critical wavelength predicted by the instability theories can grow with time. At a specific relative velocity, the perturbation of the most unstable wavelength predicted by the instability theories would grow at the highest rate.

Table 2 The fluid properties used in the calculation

\begin{tabular}{|l|c|c|c|}
\hline Properties & $\begin{array}{l}\text { Density } \\
(\rho), \mathrm{kg} / \mathrm{m}^{3}\end{array}$ & $\begin{array}{l}\text { Dynamic viscosity } \\
(\mu), \mathrm{Pa} \cdot \mathrm{s}\end{array}$ & $\begin{array}{l}\text { Surface tension } \\
(\sigma), \mathrm{N} / \mathrm{m}\end{array}$ \\
\hline Air & 1.1308 & $1.7798 \times 10^{-5}$ & 0.059 \\
\hline Water & 997.08 & $8.9307 \times 10^{-4}$ & 0. \\
\hline
\end{tabular}

A comparison between the critical and most unstable conditions predicted by different $\mathrm{KH}$ instability theories is shown in Fig. 4.

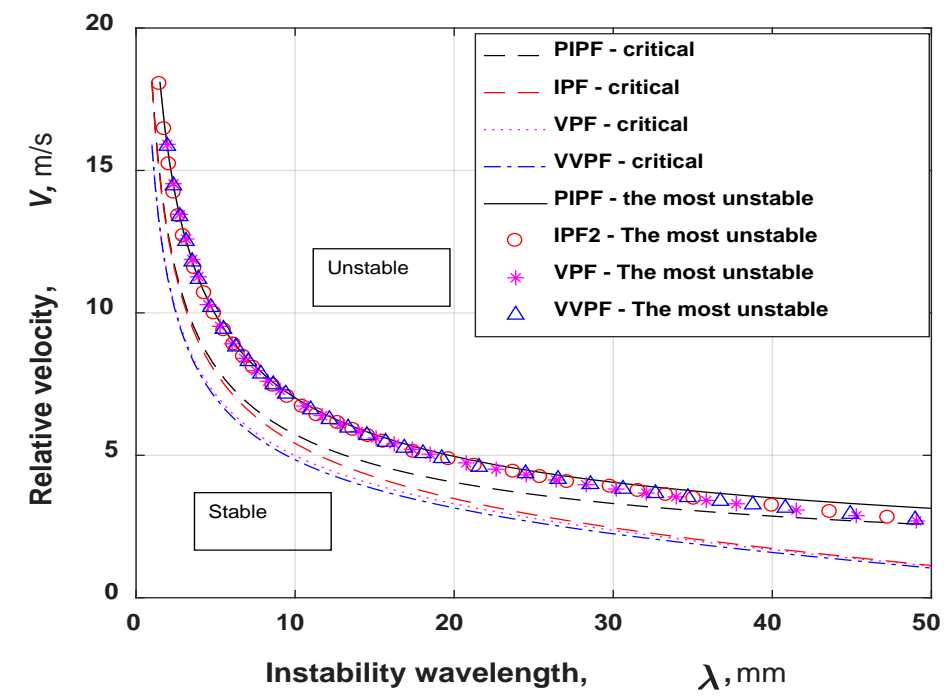

Fig. 4 Comparison of the critical and most unstable conditions predicted by different instability theories $(R i=10 \mathrm{~mm}, R c=30 \mathrm{~mm})$ 
As can be seen from Fig. 4, both VPF and VCVPF theories predict a lower relative velocity compared to the IPF theory at the critical instability conditions, so the viscosity contributes to destabilising the interface. Fig. 4 also shows that the PIPF theory commonly used in the critical heat flux model always overpredicts the required velocity at the critical conditions. As a result, the predicted critical heat flux could be an overestimation. In the most unstable conditions, Fig. 4 shows that the predictions from the IPF, VPF and VCVPF methods overlap, which indicates that the viscosity has a negligible effect in this regime. Moreover, Fig. 4 shows that the PIPF theory is a good agreement with other viscous theories for the most unstable conditions.

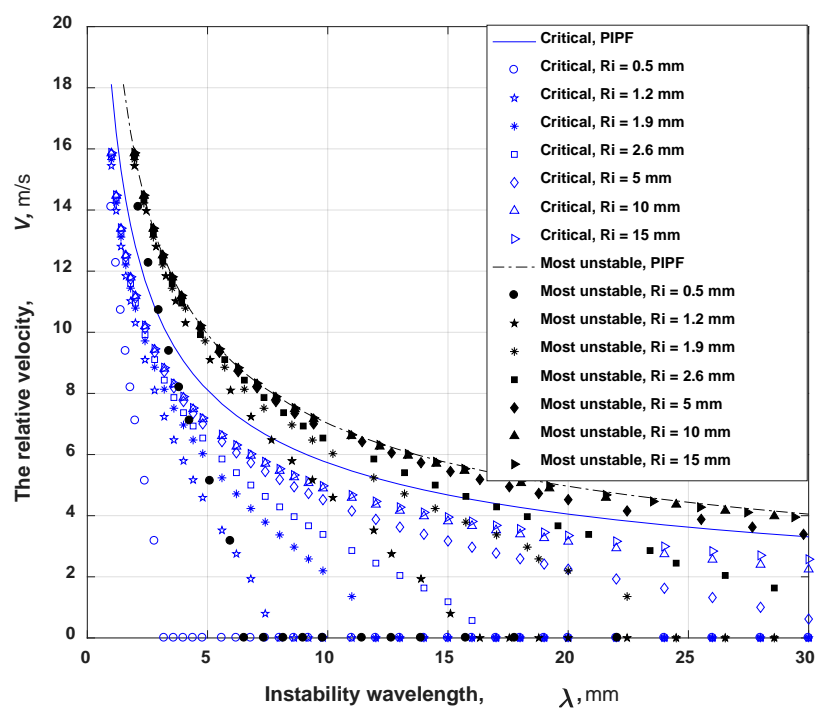

Fig. 5 The effect of the air column size on its surface instability predicted by the VCVPF theory and the experimental data

The effect of the air column size on the interface instability is shown in Fig. 5. At the critical conditions, Fig. 5 shows that the required relative velocity to trigger the instability is reduced significantly as the air column becomes smaller, especially when the radius of the air column is $<10 \mathrm{~mm}$. Fig. 5 also shows that the required relative velocity is less sensitive to the air column size when the 
dominant instability wavelength is small. Moreover, Fig. 5 suggests that there is a threshold instability wavelength, beyond which instability occurs regardless of the relative velocity. This threshold instability wavelength becomes smaller as the air column shrinks.

Fig. 5 shows that the most unstable wavelengths are always larger than the critical instability wavelength at the same relative velocity. In the most unstable condition, Fig. 5 shows that the PIPF always overpredicts the required relative velocity, especially when the radius of the gas column is $<10 \mathrm{~mm}$. When the radius of the gas column is $>10 \mathrm{~mm}$, both VCVPF and PIPF theories predict similar relative velocities. Similar to the critical condition, the required relative velocity for the most unstable conditions is insensitive to the air column size when the instability wavelength is small and the radius of the air column is $>1.2$ $\mathrm{mm}$.

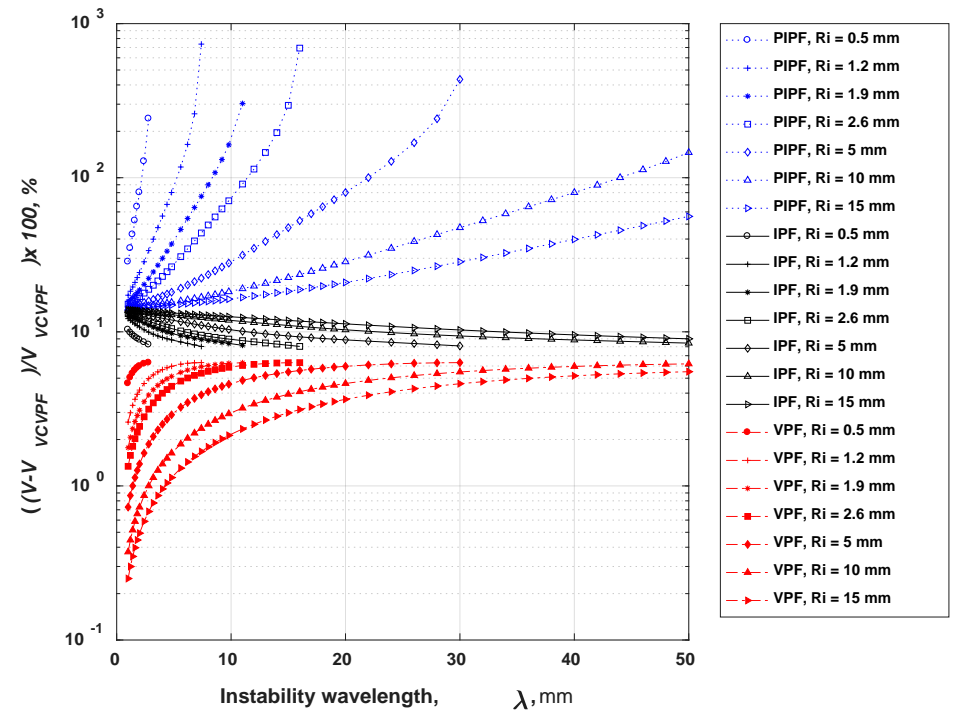

Fig. 6 Percentage difference of the critical relative velocity calculated by the IPF theory and VPF theory compared to the VCVPF theory

The percentage differences in the critical relatively velocity predicted by the IPF and VPF models, when compared to the VCVPF model, are shown in Fig. 6. 
This figure indicates that the commonly adopted PIPF theory can significantly overpredict the critical relative velocity, especially when the gas column is small. The IPF model overpredicts the relative velocity by $8-15 \%$, depending on the gas column size. In comparison, the VPF theory overpredicts the relative velocity by less than $6 \%$ in most cases.

The comparison between the measured break-up conditions and the conditions predicted by the PIPF and VCVPF models is shown in Fig. 7. The air column size can vary in the different experiments, so the $R i$ values shown in Fig. 7 are chosen to be the minimum and maximum values observed during the experiments.
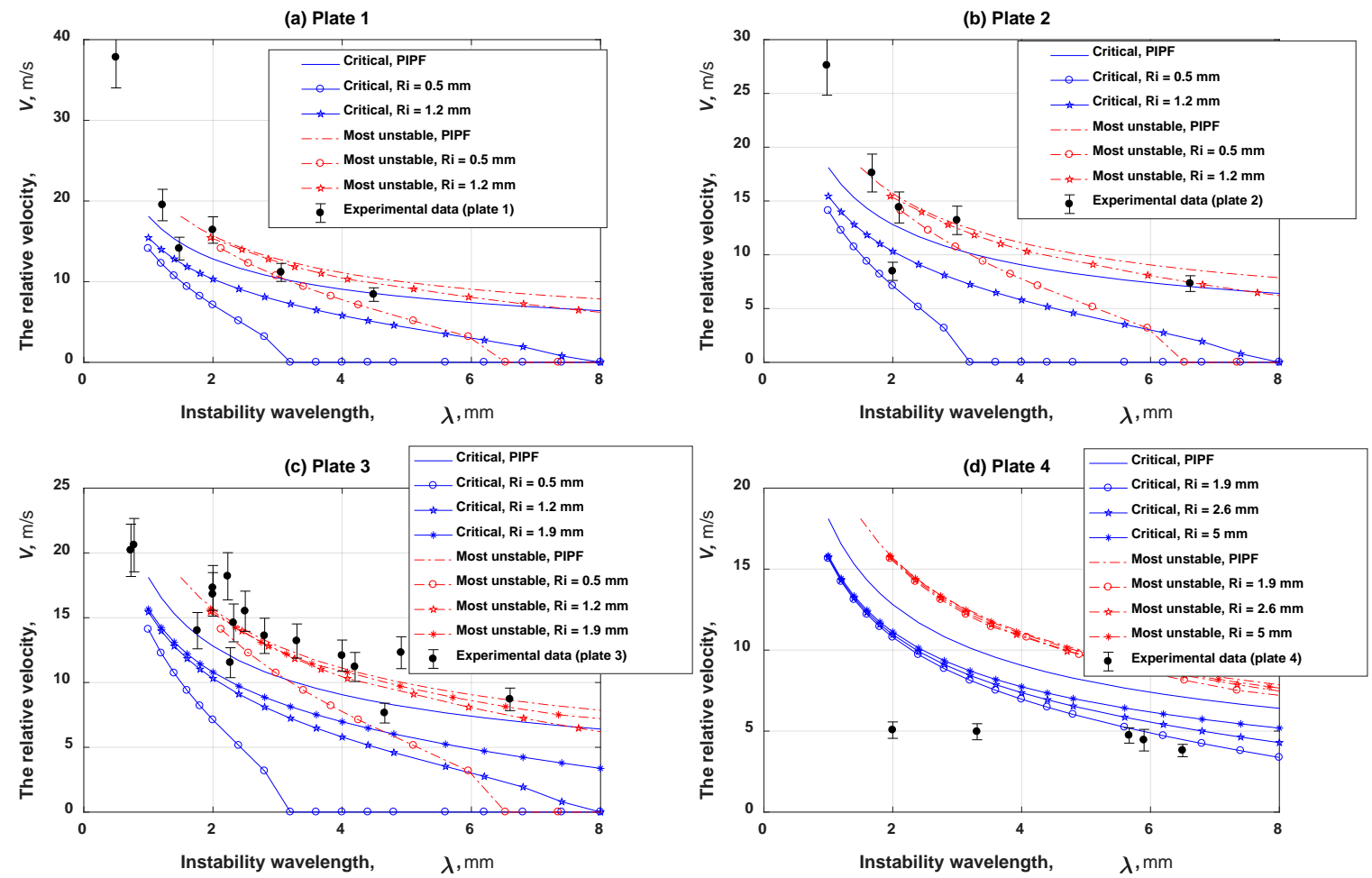

Fig. 7 Comparison of the measured air column break-up conditions in water and the instability condition predicted by the VCVPF KH model. Error bars represent a $10 \%$ uncertainty margin 
In tests using plate 1 (comprising a single $0.5 \mathrm{~mm}$ diameter hole), the majority of the air columns break-up very close to the surface due to the imposed instability on a tiny air column next to the surface $\left(h_{\text {neck }} \leq 0.5 \mathrm{~mm}\right)$, as is shown in Fig. 8 (a). The data point in the top-left coner of the plot in Fig. 7 (a) represents the typical instability wavelength and velocity. Very occasionally, the initial rapid expansion of the air column close to the surface can lead to breakup at higher positions. As can be seen from Fig. 7 (a), the most unstable conditions predicted by the VCVPF model best match the break-up conditions at higher positions. The air columns in tests using plate 2 have similar break-up patterns to those observed with plate 1, but their sizes are slightly larger.

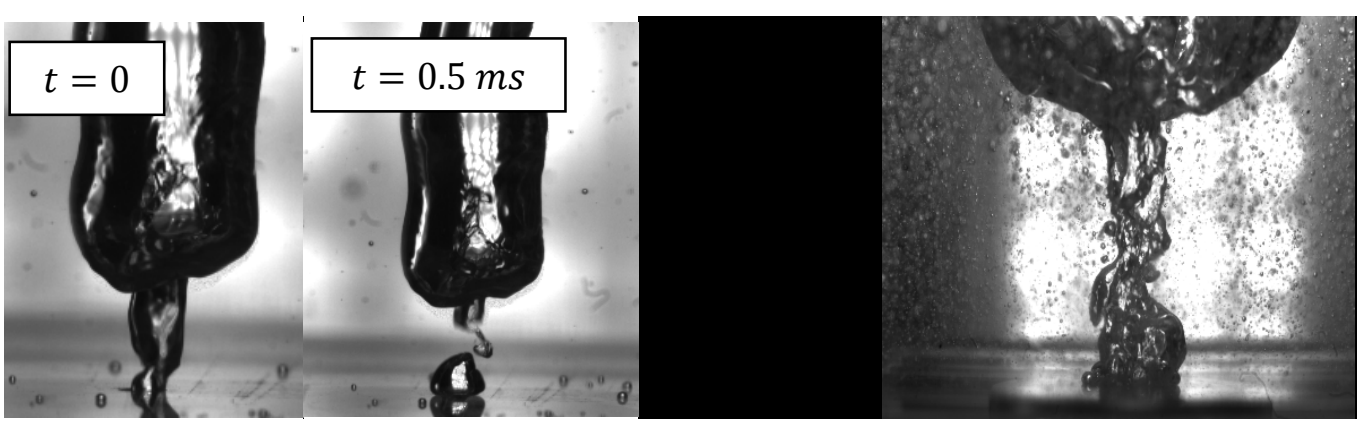

(a) Common break-up mode: plate 1

(b) Highly distorted vapour column: plate 4

Fig. 8 The typical vapour columns when using plate 1 and plate 4 In tests using plate 3 , more air columns break up at higher positions because the intensive bubble merging processes close to the surface produce a larger and more resilient air column. Fig. 7 (c) shows that the vapour columns were mostly breaking up in conditions close to the most unstable conditions predicted by both the PIPF and VCVPF models. In tests using plate 4, the air columns behaved quite differently. Fig. 7 (d) shows that the actual break-up occurs at much lower velocities than all the model predicted. Fig. 8 (b) shows a typical vapour column observed during the tests. It shows that the air column surface is 
highly distorted and the surrounding liquids are highly turbulent. Therefore, part of the reason for the larger discrepancy in the required velocity is the overestimated cross-sectional area due to the assumption of an elliptical column cross-sectional area in the reconstruction algorithm. A further reason for the discrepancy could be the oversimplified boundary conditions used in the existing VCVPF model (i.e. the use of potential flow velocity fields and neglect of the effects of gravity on the interface).

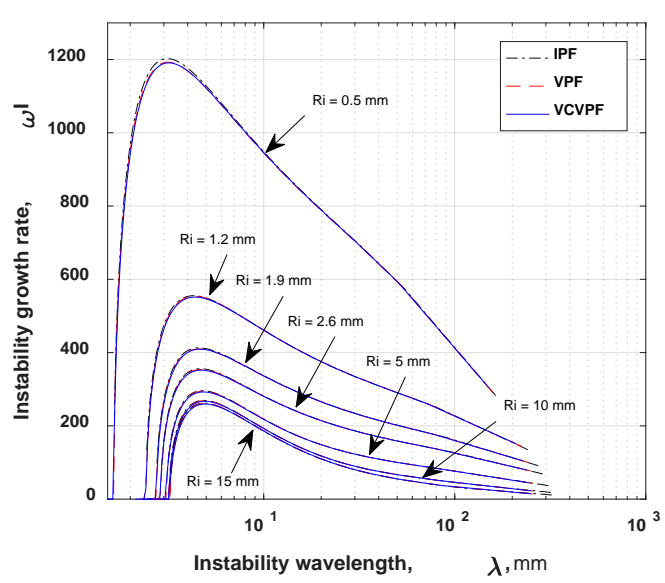

(a)

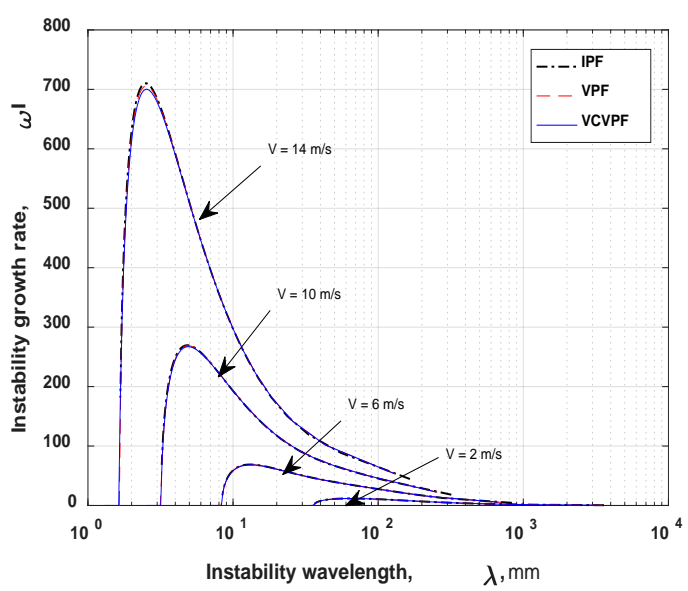

(b)

Fig. 9 The change of instability growth rates due to (a) the effect of the air column size $(V=10 \mathrm{~m} / \mathrm{s})$, and (b) the effect of relative velocity ( $R i=10$ $\mathrm{mm})$

The effect of the air column size on the instability growth rate is shown in Fig. 9 (a) which shows that the VPF and VCVPF models always predict a marginally lower instability growth rate compared to IPF models, so the viscosity helps to damp the perturbation. As the column size shrinks, the instability growth rate will increase and, as a result, the air column breaks up faster, which is consistent with the experimental observations. Fig. 9 (b) shows that the instability growth rate increases as the relative velocity increases. 
The effect of the changing water chamber size on the air-water interface instability is shown in Fig. 10, which indicates that the change of chamber size $\left(R_{c}\right)$ has a negligible effect on the required relative velocity at the critical condition when $R_{c}>1.5 R_{i}$. For the most unstable condition, the increase of the water chamber size requires a higher velocity when $R_{c} \leq 2 R_{i}$, but has a negligible effect when $R_{c}>2 R_{i}$. In addition, as the chamber becomes smaller, the discrepancy of the predicted relative velocity between the VCVPF theory and the PIPF theory becomes larger.
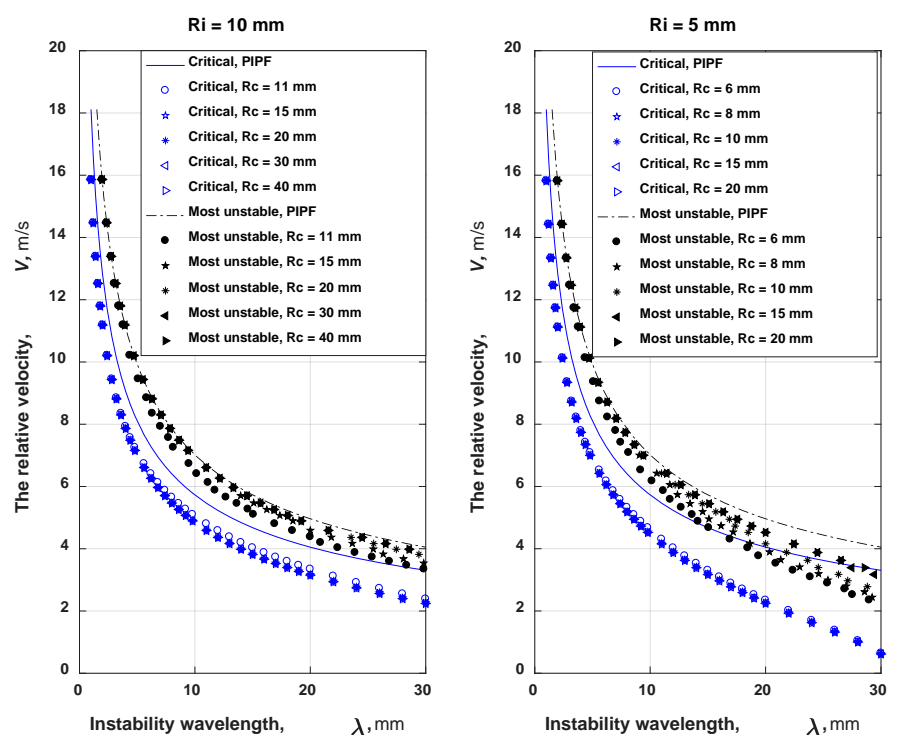

Fig. 10 The effect of the water chamber size on the air-water interface instability predicted by the VCVPF theory

\subsection{Instability of the Interface with Asymmetrical Perturbation (i.e. $m>0$ )}

As observed during the experiments, the air column is always subject to asymmetrical perturbations in practical systems. The impact of the perturbation symmetry on the critical and the most unstable conditions could be quantified by changing the constant $m$ in the instability models. The effect of changing $k$ and 
$m$ in Eq. (9) on the shape of the disturbed air column is illustrated in Fig. 11, which shows a highly distorted column at large $m$.
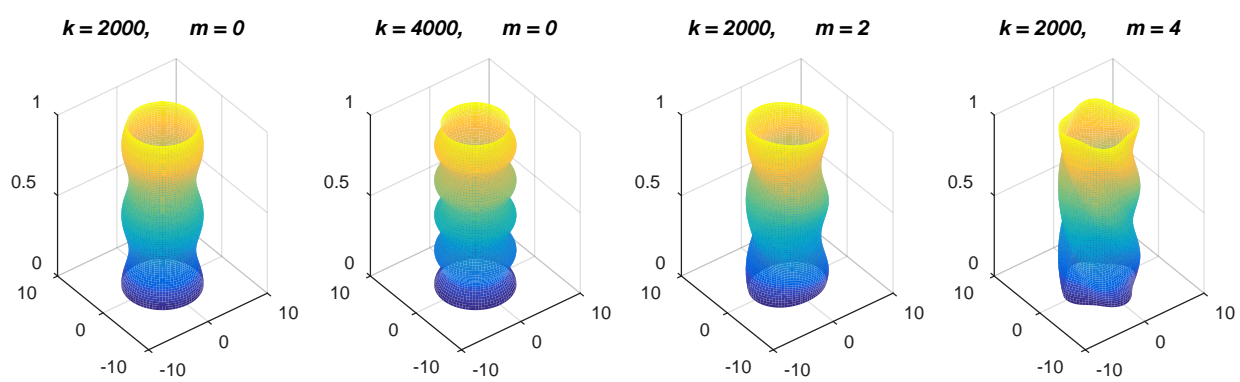

Fig. 11 Air column with different perturbations defined by Eq. (12), $R i=5, \eta_{0}=$ $0.5)$
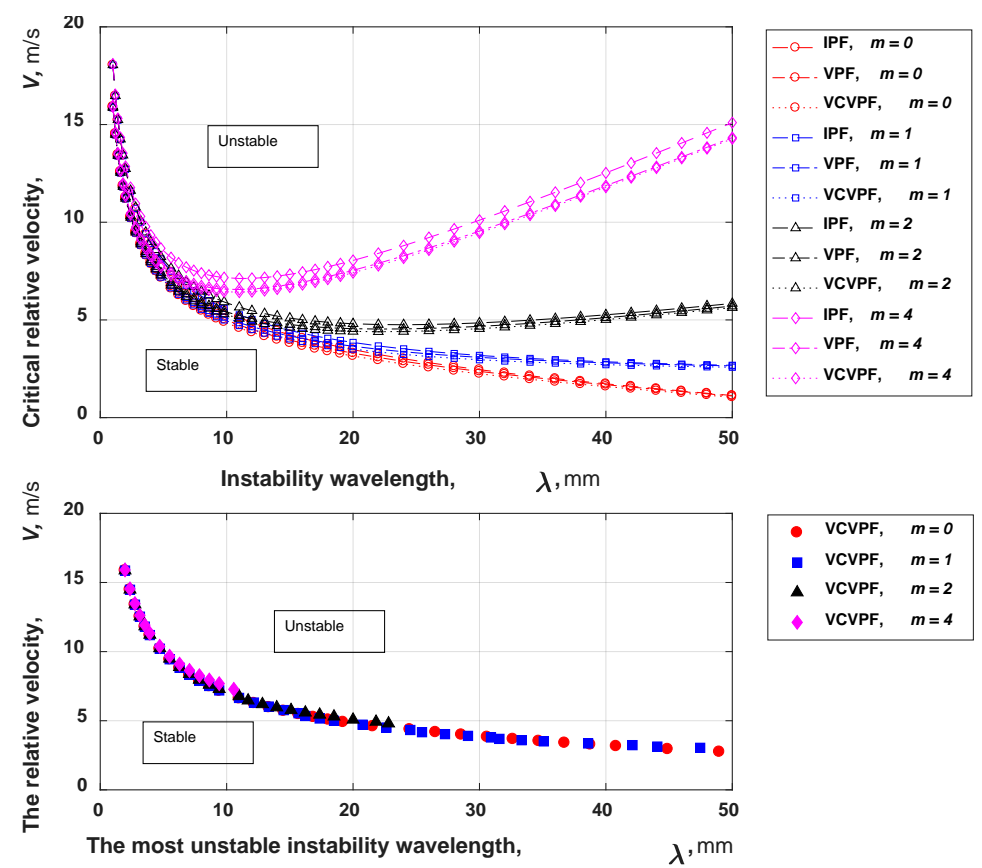

Fig. 12 The critical and most unstable conditions for air columns under axisymmetric $(m=0)$ and asymmetric $(m>0)$ perturbations, $R=$ $10 \mathrm{~mm}, R_{c}=30 \mathrm{~mm}$

The effect of the perturbation symmetry on the critical and most unstable conditions are shown in Fig. 12. This shows that the asymmetric perturbation $(m>0)$ requires a larger critical relative velocity compared to the axisymmetric perturbation at the critical conditions. However, the pertubation symmetry has a negligible effect on the most unstable condition. A very interesting observation 
from the results is the existence of a stable region for all instability wavelengths when $m \geq 2$. This region is defined by a threshold relative velocity that corresponds to the minimum relative velocity for both the critical and the most unstable conditions. When then relative velocity is smaller than this threshold value, the air column is always stable. The threshold relative velocity increases as the perturbation becomes more asymmetric (i.e. larger $m$ ). This implies that in applications affected by the instability of gas columns, controlling the pertubation symmetry by adjusting the liquid flow could be a potential way to generate highly stable gas columns. The most unstable conditions calculated from the IPF and VPF theories are very close to the conditions calculated by the VCVPF theory and are thus not shown here.

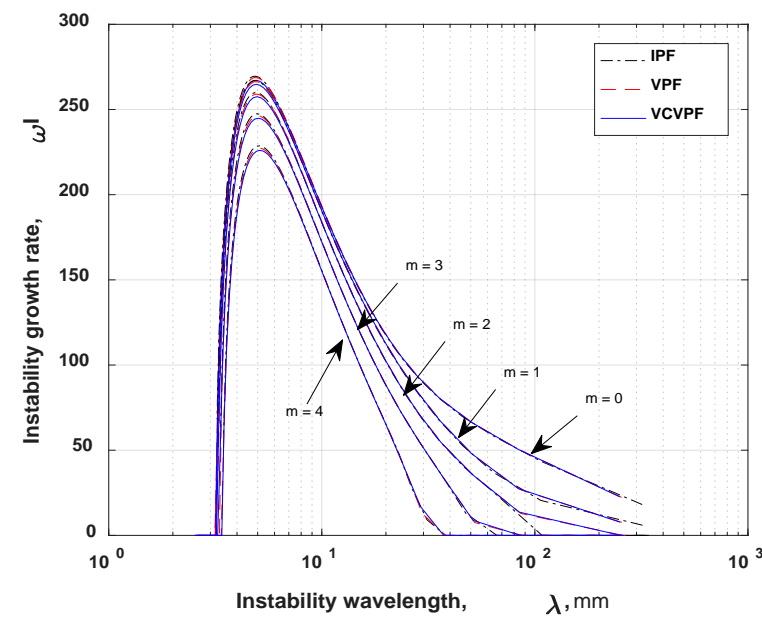

Fig. 13 The instability growth rate for air columns under axisymmetric $(m=0)$ and asymmetric $(m>0)$ perturbations, $V=10 \mathrm{~m} / \mathrm{s}$

The effect of perturbation symmetry on the instability growth rate is shown in Fig. 13. It shows that the asymmetric perturbation has a smaller growth rate compared to the axisymmetric one and the VCVPF theory always predicts a marginally smaller instability growth rate compared to the IPF and VPF theories.

\section{Conclusion and Future Work}


In this paper, the Kelvin-Helmholtz instability conditions for a cylindrical interface between two viscous immiscible fluids with finite depths have been investigated both theoretically and experimentally. Following the established IPF, VPF and VCVPF analysis approaches reported in the literature, but using different boundary conditions, the critical condition (neutral curve) has been derived analytically, and the most unstable conditions have been calculated numerically. The comparisons between the predictions of different models, the measured break-up conditions and parametric analysis of the different models show the following significant conclusions:

(1) The viscosity reduces the required relative velocities at the critical instability conditions, but has negligible effects on the most unstable conditions.

(2) The relative velocity required to trigger the critical instability condition is reduced significantly as the radius of the cylindrical interface falls below $10 \mathrm{~mm}$. Moreover, there is a threshold instability wavelength, above which the instability occurs regardless of the relative velocity. This threshold value reduces as the air column shrinks.

(3) The required relative velocity at the most unstable condition is insensitive to the size of the cylindrical interface when the interface radius is $>1.2$ $\mathrm{mm}$.

(4) The most unstable conditions predicted by the VCVPF model match well with the measured air columns break-up conditions in water when the interface is not exposed to highly turbulent flows. 
(5) Both the critical and most unstable conditions are insensitive to the chamber size as long as the chamber radius is greater than twice the radius of the cylindrical interface.

(6) The perturbation symmetry has a noticeable effect on the critical conditions but has a negligible effect on the most unstable conditions.

The newly developed and validated viscous $\mathrm{KH}$ instability models presented in this paper aim to improve the prediction of the instability conditions of the cylindrical interface shown in Fig. 1 (c), where there is negligible interfacial heat and mass transfer, such as in saturated boiling. In conditions where there is a noticeable temperature gradient across the cylindrical interface, such as in subcooled boiling, more detailed theoretical and experimental investigations into the interfacial heat and mass transfers need to be conducted. One of the challenges when conducting a detailed heat transfer analysis on the configuration shown in Fig. 1 (c) is the lack of an axisymmetric heating source. As a result, a transient multi-dimensional heat transfer analysis will need to be carried out to calculate the time- and space-dependent temperature and heat flux profiles. Therefore, a unified transient heat/mass transfer model and a viscous instability model are likely to require a numerical model. Moreover, the effects of turbulence and gravity on a highly distorted interface need further investigation.

\section{Acknowledgements}

We would like to thank Dr Vitaliy Sechenyh for his kind support in our experimental data analysis, especially for providing the useful codes to do the 
reconstruction. We would also like to thank Dr Francois Nadal and Prof Colin Garner for their advice and support in revising the manuscript. 


\section{References:}

Asthana, R. and Agrawal, G. S., 2007. Viscous potential flow analysis of KelvinHelmholtz instability with mass transfer and vaporization. Physica A: Statistical Mechanics and its Applications, 382(2), 389-404. doi:

10.1016/j.physa.2007.04.037.

Asthana, R., Awasthi, M. K. and Agrawal, G. S., 2014. Viscous Potential Flow Analysis of Kelvin-Helmholtz Instability of a Cylindrical Flow with Heat and Mass Transfer. Heat Transfer-Asian Research, 43(6), 489-503. doi: 10.1002/htj.21092.

Awasthi, M. K. and Agrwal, G. S., 2011. Viscous potential flow analysis of Kelvin-Helmholtz instability of cylindrical interface. International Journal of Applied Mathematics and Computation, 3(2), 131-140. doi:

10.0000/ijamc.2011.3.2.271.

Awasthi, M. K., Asthana, R. and Agrawal, G. S., 2012. Pressure corrections for the potential flow analysis of Kelvin-Helmholtz instability with heat and mass transfer. International Journal of Heat and Mass Transfer. Elsevier Ltd, 55(910), 2345-2352. doi: 10.1016/j.ijheatmasstransfer.2012.01.032.

Awasthi, M. K., Asthana, R. and Agrawal, G. S., 2014. Viscous correction for the viscous potential flow analysis of Kelvin-Helmholtz instability of cylindrical flow with heat and mass transfer. International Journal of Heat and Mass Transfer. Elsevier Ltd, 78(6), 251-259. doi: 10.1016/j.ijheatmasstransfer.2014.06.082.

Awasthi, M. K., Asthana, R. and Uddin, Z., 2016. Nonlinear Study of KelvinHelmholtz instability of cylindrical flow with mass and heat transfer. International Communications in Heat and Mass Transfer. Elsevier Ltd, 71, 216-224. doi: 10.1016/j.icheatmasstransfer.2015.12.035.

Carey, V. P., 2008. Liquid-vapor phase change phenomena. 2nd Edition. CRC Press, New York.

Funada, T. and Joseph, D. D., 2001. Viscous potential flow analysis of KelvinHelmholtz instability in a channel. Journal of Fluid Mechanics. Loughborough University, 445(May), 263-283. doi: 10.1017/S0022112001005572.

Funada, T. and Joseph, D. D., 2002. Viscous potential flow analysis of capillary instability. International Journal of Multiphase Flow, 28(9), 1459-1478. doi: 10.1016/S0301-9322(02)00035-6.

Hsieh, D. Y., 1978. Interfacial stability with mass and heat transfer. Physics of Fluids, 21(8), 745-748. doi: 10.1063/1.862763.

Joseph, D. D. and Wang, J., 2004. The dissipation approximation and viscous potential flow. Journal of Fluid Mechanics, 505, 365-377. doi: 10.1017/S0022112004008602.

Lee, D.-S., 2007. Nonlinear Kelvin-Helmholtz instability of cylindrical flow with 
mass and heat transfer. Physica Scripta, 76(1), 97-103. doi: 10.1088/00318949/76/1/017.

Nayak, A. R. and Chakraborty, B. B., 1984. Kelvin-Helmholtz stability with mass and heat transfer. Physics of Fluids Phys. Fluids Fluids Fluids, 27(8), 19371941. doi: 10.1063/1.1706875.

Sechenyh, V., Turner, J., Sykes, D., Duke, D. J., Swantek, A. B., Matusik, K. E., Kastengren, A. L., Powell, C. F., Viera, A., Payri, P. and Crua, C., 2017.

Quantification of diesel injector dribble using 3D reconstruction from X-ray and DBI imaging. In 28th Conference on Liquid Atomization and Spray Systems. doi: http://dx.doi.org/10.4995/ILASS2017.2017.4742.

Wang, J., Joseph, D. D. and Funada, T., 2005. Viscous contributions to the pressure for potential flow analysis of capillary instability of two viscous fluids', Physics of Fluids, 17(5), 1-12. doi: 10.1063/1.1914573.

Zhao, H. and Williams, A., 2018. Predicting the critical heat flux in pool boiling based on hydrodynamic instability induced irreversible hot spots. International Journal of Multiphase Flow. doi: 10.1016/j.ijmultiphaseßow.2018.02.021. 


\section{Appendix 1 Derivation of the linear instability equations}

With a disturbance, the interface is represented by Eq. (A1.1).

$S(r, t)=r-R-\eta=0$

where $\eta$ is the displacement of the interface caused by the perturbation.

Assuming both fluid 1 and fluid 2 experience irrotational flow, their velocity potentials should satisfy the Laplace equation, i.e. Eq. (A1.2).

$\nabla^{2} \phi_{i}=0 ; \boldsymbol{u}_{i}=\nabla \phi_{i} ;(i=1,2)$

where $i=1$ refers to fluid 1 and $i=2$ refers to fluid 2 .

The fluids are considered to flow with uniform velocity $U_{1}$ and $U_{2}$, respectively along the $z$-axis. Therefore, in the initial state, the potential functions can be written as:

$\phi_{i}^{0}=U_{i} z,(i=1,2)$

In cases where fluid 1 and fluid 2 are immiscible, the conservation of mass across the interface is given as follows:

$\llbracket \rho\left(\frac{\partial S}{\partial t}+\nabla \phi \cdot \nabla S\right) \rrbracket=0$

where $\llbracket A \rrbracket$ represents the difference in any quantity $A$ across the interface, i.e. $\llbracket A \rrbracket=A_{2}-A_{1}$.

The conservation of momentum across the vertical interface with negligible gravity is given by: 
$\llbracket \rho(\nabla \phi \cdot \nabla S) \times\left(\frac{\partial S}{\partial t}+\nabla \phi \cdot \nabla S\right)+p \rrbracket=-\sigma \nabla^{2} \eta$

where $p$ is the pressure due to the viscous forces; $\sigma$ is the surface tension between fluid 1 and fluid 2 .

When there is a phase change of fluid 1 across the interface, and there is a negligible change of sensible heat, the conservation of energy across the interface can be written as:

$h_{f g} \rho_{1}\left(\frac{\partial S}{\partial t}+\nabla \phi_{1} \cdot \nabla S\right)=Q(\eta)$

where $Q(\eta)$ is the net heat flux across the boundary; $h_{f g}$ is the latent heat when fluid is transformed from phase 1 to phase 2 .

The exact expression to describe the heat flux should be derived from the heat transfer equation based on the specified boundary conditions. This paper will, however, focus on conditions where there is negligible heat and mass transfer across the interface, i.e. $Q(\eta)=0$, so the energy equation will be bypassed.

The boundary conditions are summarised as follows:

(3) The rigid chamber wall: $\frac{\partial \phi_{2}}{\partial r}=0$ at $r=b$.

(4) Finite value of the velocity in the central axis: $\frac{\partial \phi_{1}}{\partial r}<\infty$ at $r=0$.

The normal mode analysis is used to solve the above equations by considering the interface disturbance displacement:

$\eta=\eta_{0} \cdot \exp (i(k z+m \theta-w t))+c . c$. 
where $\eta_{0}$ is the disturbance amplitude; $i=\sqrt{-1} ; k$ is the instability wavenumber and is correlated to the instability wavelength $(\lambda)$ by $k=2 \pi / \lambda ; w=w_{r}+i w_{i}$ is the complex growth rates; c.c. stands for the complex conjugate.

The first-order solution of Eq. (A1.2) in cylindrical coordinates is given by:

$\phi_{1}^{1}=\left(A \cdot I_{m}(k r)+B \cdot K_{m}(k r)\right) \cdot \exp (i(k z+m \theta-w t))+c . c$.

$\phi_{2}^{1}=\left(C \cdot I_{m}(k r)+D \cdot K_{m}(k r)\right) \cdot \exp (i(k z+m \theta-w t))+c . c$.

where $A, B, C, D$ are coefficients that need to found;

$I_{m}$ is the modified Bessel function of the first kind;

$K_{m}$ is the modified Bessel function of the second kind;

$m$ is the symmetric index along the $\theta$ direction; $m=0$ corresponds to the axisymmetric disturbance.

By solving Eqs. (A1.4), (A1.10) and (A1.11) together with the boundary conditions defined by Eqs. (A1.7) and (A1.8), the following solutions for the velocity potentials are obtained:

$\phi_{1}^{1}=\frac{\eta_{0}}{k}\left(i\left(U_{1} k-w\right)\right) \cdot F_{1}(k r) \cdot \exp (i(k z+m \theta-w t))+c . c$.

$\phi_{2}^{1}=\frac{\eta_{0}}{k}\left(i\left(U_{2} k-w\right)\right) \cdot E_{1}(k r) \cdot \exp (i(k z+m \theta-w t))+c . c$.

where $F_{1}(k r)=\frac{I_{m}(k r)}{I_{m}^{\prime}(k R)}, E_{1}(k r)=\frac{I_{m}(k r) K_{m}^{\prime}(k b)-K_{m}(k r) I_{m}^{\prime}(k b)}{I_{m}^{\prime}(k R) K_{m}^{\prime}(k b)-I_{m}^{\prime}(k b) K_{m}^{\prime}(k R)} ; I_{m}^{\prime}(k R), K_{m}^{\prime}(k R)$ are the first-order derivatives of $I_{m}(k r)$ and $K_{m}(k r)$ at $r=R$ respectively. 
Different assumptions have been made and different approaches have been developed to calculate the viscous pressure term (i.e. $p$ in Eq. (A1.5)). This is now discussed.

\section{Inviscid Potential Flow (IPF) Analysis}

The IPF ignores the viscous pressure term, so $p$ in Eq. (A1.5) equals zero.

Based on Eqs. (A1.5) and (A1.12), the following dispersion equation can be derived:

$a_{0} w^{2}+a_{1} w+a_{2}=0$

where $a_{0}=\rho_{2} E_{1 R}-\rho_{1} F_{1 R} ; a_{1}=2 k\left(U_{1} \rho_{1} F_{1 R}-U_{2} \rho_{2} E_{1 R}\right)$;

$a_{2}=k^{2}\left(U_{2}^{2} \rho_{2} E_{1 R}-U_{1}^{2} \rho_{1} F_{1 R}\right)+\frac{k \sigma}{R^{2}}\left(k^{2} R^{2}-1+m^{2}\right) ;$

$E_{1 R}=E_{1}(k R) ; F_{1 R}=F_{1}(k R)$.

\section{Viscous Potential Flow (VPF) Analysis}

The VPF model takes into account the pressure term caused by the normal viscous force. For Newtonian fluids, the viscous pressure term can be represented as:

$p_{i}=2 \mu_{i}\left(\frac{\partial^{2} \phi_{i}^{1}}{\partial r^{2}}\right) ; i=1,2$

where $\mu$ is the dynamic viscosity.

Based on Eqs. (A1.5), (A1.12) and (A1.14), the following dispersion equation can be derived:

$a_{0} w^{2}+\left(a_{1}+i b_{1}\right) w+\left(a_{2}+i b_{2}\right)=0$ 
where $a_{0}=\rho_{2} E_{1 R}-\rho_{1} F_{1 R} ; a_{1}=2 k\left(U_{1} \rho_{1} F_{1 R}-U_{2} \rho_{2} E_{1 R}\right)$;

$b_{1}=2 k^{2}\left(\mu_{2} E_{2 R}-\mu_{1} F_{2 R}\right) ; a_{2}=k^{2}\left(U_{2}^{2} \rho_{2} E_{1 R}-U_{1}^{2} \rho_{1} F_{1 R}\right)+\frac{k \sigma}{R^{2}}\left(k^{2} R^{2}-1+m^{2}\right) ;$

$b_{2}=2 k^{3}\left(\mu_{1} U_{1} F_{2 R}-\mu_{2} U_{2} E_{2 R}\right)$

$E_{2 R}=E_{1 \mathrm{R}}\left(1+\frac{m^{2}}{k^{2} R^{2}}\right)-\frac{1}{k R} ; F_{2 R}=F_{1 \mathrm{R}}\left(1+\frac{m^{2}}{k^{2} R^{2}}\right)-\frac{1}{k R}$

\section{Viscous Correction for the Viscous Potential Flow (VCVPF) Analysis}

The VCVPF theory corrects the VPF theory by including the extra term for the viscous shear stress so the viscous pressure term $(p)$ can be written as:

$p_{i}=2 \mu_{i}\left(\frac{\partial^{2} \phi_{i}^{1}}{\partial r^{2}}\right)-p_{i}^{v} ; i=1,2$

where $p_{i}^{v}$ is the shear stress term and has been shown by Wang et al. (2005) to satisfy the Laplace equation in the linear mode analysis:

$\nabla^{2} p_{i}^{v}=0$

Based on Eqs. (A1.5), (A1.12) and (A1.17), the following dispersion equation can be derived:

$a_{0} w^{2}+\left(a_{1}+i b_{1}\right) w+\left(a_{2}+i b_{2}\right)=0$

where $a_{0}=\rho_{2} E_{1 R}-\rho_{1} F_{1 R} ; a_{1}=2 k\left(U_{1} \rho_{1} F_{1 R}-U_{2} \rho_{2} E_{1 R}\right)$;

$b_{1}=2 k^{2}\left[\mu_{2} E_{3 R}-\mu_{1} F_{3 R}\right] ; a_{2}=k^{2}\left(U_{2}^{2} \rho_{2} E_{1 R}-U_{1}^{2} \rho_{1} F_{1 R}\right)+\frac{k \sigma}{R^{2}}\left(k^{2} R^{2}-1+m^{2}\right) ;$

$b_{2}=2 k^{3}\left[\mu_{1} U_{1} F_{3 R}-\mu_{2} U_{2} E_{3 R}\right] ; E_{3 R}=E_{1 R}+E_{2 R} ; F_{3 R}=F_{1 R}+F_{2 R}$

The neutral curve can be found based on the condition: $a_{0} b_{2}^{2}-a_{1} b_{1} b_{2}+a_{2} b_{1}^{2}=$ 0, as in Eq. (A1.19). 


$$
V_{V C V P F}^{2}=\left(U_{1}-U_{2}\right)^{2}=\frac{\left[\sigma k\left(k^{2} R^{2}+m^{2}-1\right) / R^{2}\right] \cdot\left[2 k^{2}\left(\mu_{1} F_{3 R}-\mu_{2} E_{3 R}\right)\right]^{2}}{4 k^{6}\left(\rho_{1} \mu_{2}^{2} F_{1 R} E_{3 R}^{2}-\rho_{2} \mu_{1}^{2} E_{1 R} F_{3 R}^{2}\right)}
$$

\section{Figure Captions}

Fig. 1 Different geometries of interfaces and boundary conditions reported in the literature

Fig. 2 Schematic experimental setup of the vapour column instability rig

Fig. 3 The air column necking process shown from two orthogonal angles

Fig. 4 Comparison of the critical and most unstable conditions predicted by different instability theories $(R i=10 \mathrm{~mm}, R c=30 \mathrm{~mm})$

Fig. 5 The effect of the air column size on its surface instability predicted by the VCVPF theory and the experimental data

Fig. 6 Percentage difference of the critical relative velocity calculated by the IPF theory and VPF theory compared to the VCVPF theory

Fig. 7 Comparison of the measured air column break-up conditions in water and the instability condition predicted by the VCVPF KH model. Error bars represent $10 \%$ uncertainty margin

Fig. 8 The typical vapour columns when using plate 1 and plate 4

Fig. 9 The change of instability growth rates due to (a) the effect of the air column size $(V=10 \mathrm{~m} / \mathrm{s})$, and (b) the effect of relative velocity $(R i=10$ $\mathrm{mm})$

Fig. 10 The effect of the water chamber size on the air-water interface instability predicted by the VCVPF theory

Fig. 11 Air column with different perturbations defined by Eq. (12), $R i=5, \eta_{0}=$ $0.5)$

Fig. 12 The critical and most unstable conditions for air columns under axisymmetric $(m=0)$ and asymmetric $(m>0)$ perturbations, $R=$ $10 \mathrm{~mm}, R_{c}=30 \mathrm{~mm}$

Fig. 13 The instability growth rate for air columns under axisymmetric $(m=0)$ and asymmetric $(m>0)$ perturbations, $V=10 \mathrm{~m} / \mathrm{s}$

\section{List of Tables}

Table 2 Summary of experimental conditions

Table 2 The fluid properties used in the calculation 